\title{
Helping Seniors Plan for Posthospital Discharge Needs Before a Hospitalization Occurs: Results from the Randomized Control Trial of PlanYourLifespan.org
}

Lee A. Lindquist, MD, MPH, MBA ${ }^{1 *}$, Vanessa Ramirez-Zohfeld, MPH${ }^{1}$, Priya D. Sunkara, MA¹, Chris Forcucci, RN, BSN²,

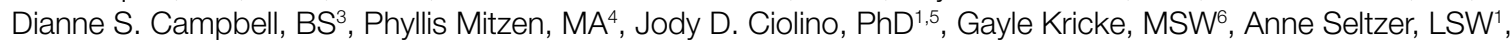
Ana V. Ramirez, BA ${ }^{7}$, Kenzie A. Cameron, PhD, MPH ${ }^{1,5,8}$

\begin{abstract}
${ }^{1}$ Division of General Internal Medicine and Geriatrics, Department of Medicine, Northwestern University Feinberg School of Medicine, Chicago, Illinois; ${ }^{2}$ Aging and In-Home Services of Northeast Indiana, Fort Wayne, Indiana; ${ }^{3}$ The Village Chicago, Chicago, Illinois; ${ }^{4}$ Skyline Village Chicago, Chicago, Illinois; ${ }^{5}$ Department of Preventive Medicine, Northwestern University Feinberg School of Medicine, Chicago, Illinois; ${ }^{6}$ Center for Healthcare Studies, Northwestern University Feinberg School of Medicine, Chicago, Illinois; 'Dartmouth College, Hanover, New Hampshire; ${ }^{8}$ Department of Medical Social Sciences, Northwestern University Feinberg School of Medicine, Chicago, Illinois.
\end{abstract}

BACKGROUND: Hospitalized seniors are frequently too sick to make informed decisions about their postdischarge care. Subsequently, loved ones often make support choices (eg, skilled nursing facility placement, caregivers) at discharge. We sought to advance the timeline for postacute care decisions to before a hospitalization occurs.

OBJECTIVE: Investigate the effect of PlanYourLifespan.org (PYL) on knowledge of posthospital discharge options.

DESIGN: Multisite randomized controlled trial.

SETTING/PATIENTS: Nonhospitalized adults, aged $\geq 65$ years, living in urban, suburban, and rural areas of Texas, Illinois, and Indiana.

INTERVENTION: PYL is a national, publicly available tool that provides education on posthospital therapy choices and local home-based resources.

MEASUREMENTS: Participants completed an in-person baseline survey, followed by exposure to intervention or attention control (AC) websites, then 1-month and 3-month telephone surveys. The primary knowledge outcome was measured with 6 items (possible 0-6 points) pertaining to hospital discharge needs.

RESULTS: Among 385 participants randomized, mean age was 71.9 years (standard deviation 5.6 ) and $79.5 \%$ of participants were female. At 1 month, the intervention group had a 0.6 point change (standard deviation $=1.6$ ) versus the AC group who had a -0.1 point change in knowledge score. Linear mixed modeling results suggest sex, health literacy level, level of education, income, and history of high blood pressure/kidney disease were significant predictors of knowledge over time. Controlling for these variables, treatment effect remained significant $(P<0.0001)$.

CONCLUSION: Seniors who used PYL demonstrated an increased understanding of posthospitalization and home services compared to the control group.

TRIAL REGISTRATION: Clinicaltrials.gov Identifier: NCTO 2256072. Journal of Hospital Medicine 2017;12:911-917. Published online first August 23, 2017. (C) 2017 Society of Hospital Medicine
When seniors are discharged from the hospital, many will require additional support and therapy to regain their independence and return safely home. ${ }^{1,2}$ Most seniors do not understand what their support needs will entail or the differences between therapy choices. ${ }^{3}$ To complicate the issue, seniors are often incapacitated and unable to make discharge selections for themselves.

Consequently, discharge planners and social workers often explain options to family members and loved ones, who frequently feel overwhelmed., ${ }^{4,5}$ While often balancing jobs, loved ones are divided between wanting to stay with

\footnotetext{
*Address for correspondence and reprint requests: Lee A. Lindquist, MD, MPH, MBA, Associate Professor of Medicine, Division of General Internal Medicine and Geriatrics, Northwestern University, Feinberg School of Medicine, 750 N. Lake Shore Drive, 10th floor, Chicago, IL 60611; Telephone: 312-503-6400; Fax: 312-503-2777; E-mail: LAL425@northwestern.edu
}

Received: January 12, 2017; Revised: May 5, 2017; Accepted: May 15, 2017 2017 Society of Hospital Medicine DOI 10.12788/jhm.2798 the senior in the hospital and driving to area skilled nursing facilities (SNFs) for consideration. Discharges are delayed waiting for families to make visits and choose an SNF. Longer lengths of stay are detrimental to seniors due to the increased risks of infection, functional loss, and cognitive decline. $^{6}$

Although seniors comprised only $12 \%$ of the US population in $2003,{ }^{7}$ they accounted for one-third of all hospitalizations, over 13.2 million hospital stays. ${ }^{8}$ Hospital stays for seniors resulted in hospital charges totaling nearly $\$ 329$ billion, or $43.6 \%$ of national hospital bills in $2003 .{ }^{7}$ Seniors are also high consumers of postacute care services. By 2050, the number of individuals using long-term care services in any setting (eg, at home, assisted living, or SNFs) will be close to 27 million. ${ }^{9-11}$ With the knowledge that many seniors will be hospitalized and subsequently require services thereafter, we sought to assist seniors in planning for their hospital discharge needs before they were hospitalized.

Our team developed PlanYourLifespan.org (PYL) to facilitate this planning for postdischarge needs and fill the 
TABLE 1. Participant Baseline Characteristics

\begin{tabular}{|c|c|c|c|c|c|}
\hline \multirow[b]{3}{*}{ Characteristics } & \multicolumn{4}{|c|}{ Treatment Arm } & \multirow[b]{3}{*}{$P$ Value } \\
\hline & \multicolumn{2}{|c|}{ Attention Control } & \multicolumn{2}{|c|}{ PLAN YOUR LIFESPAN } & \\
\hline & $N=191$ & $\%$ & $N=194$ & $\%$ & \\
\hline Mean Age ( \pm SD) & \multicolumn{2}{|c|}{$72.1(5.6)$} & \multicolumn{2}{|c|}{$71.7(5.6)$} & .51 \\
\hline Sex & & & & & .19 \\
\hline Female & 157 & 82.2 & 149 & 76.8 & \\
\hline Male & 34 & 17.8 & 45 & 23.2 & \\
\hline Race & & & & & .30 \\
\hline White & 125 & 65.4 & 117 & 60.3 & \\
\hline Non-white & 66 & 34.6 & 77 & 39.7 & \\
\hline Marital Status & & & & & .58 \\
\hline Single, never married & 27 & 14.1 & 23 & 11.9 & \\
\hline Married & 75 & 39.3 & 85 & 43.8 & \\
\hline Widowed & 44 & 23.0 & 36 & 18.5 & \\
\hline Divorced/separated & 45 & 23.6 & 50 & 25.8 & \\
\hline How would you rate your health? & & & & & .78 \\
\hline Poor & 4 & 2.1 & 4 & 2.1 & \\
\hline Fair & 22 & 11.5 & 18 & 9.3 & \\
\hline Good & 76 & 39.8 & 81 & 41.7 & \\
\hline Very Good & 62 & 32.5 & 70 & 36.1 & \\
\hline Excellent & 27 & 14.1 & 21 & 10.8 & \\
\hline Household Income & & & & & .90 \\
\hline Less than $\$ 20,000$ & 45 & 23.6 & 43 & 22.2 & \\
\hline$\$ 20,000-\$ 40,000$ & 50 & 26.2 & 54 & 27.8 & \\
\hline$\$ 40,001-\$ 60,000$ & 31 & 16.2 & 27 & 13.9 & \\
\hline$\$ 60,001-\$ 80,000$ & 25 & 13.1 & 26 & 13.4 & \\
\hline$\$ 80,001-\$ 100,000$ & 19 & 10.0 & 17 & 8.8 & \\
\hline More than $\$ 100,000$ & 13 & 6.8 & 18 & 9.3 & \\
\hline Don't Know & 4 & 2.1 & 2 & 1.0 & \\
\hline Prefer not to say & 4 & 2.1 & 7 & 3.6 & \\
\hline Education & & & & & .57 \\
\hline High school or less & 33 & 17.3 & 40 & 20.6 & \\
\hline Some college & 55 & 28.8 & 59 & 30.4 & \\
\hline College graduate & 103 & 53.9 & 95 & 49.0 & \\
\hline REALM Score & & & & & .85 \\
\hline Third grade and below & 1 & 0.5 & 0 & 0.0 & \\
\hline Fourth to sixth grade & 2 & 1.1 & 1 & 0.5 & \\
\hline Seventh to eighth grade & 29 & 15.2 & 28 & 14.4 & \\
\hline High school & 159 & 83.3 & 165 & 85.1 & \\
\hline Have you or a member of your household been hospitalized in the past 3 years? & & & & & .31 \\
\hline No & 104 & 54.5 & 96 & 49.5 & \\
\hline Yes & 86 & 45.0 & 98 & 50.5 & \\
\hline Don't know & 1 & 0.5 & 0 & 0.00 & \\
\hline
\end{tabular}

knowledge gap in understanding postdischarge options. With funding from the Patient Centered Outcomes Research Institute, we aimed to test the effectiveness of PYL on improving knowledge of hospital discharge resources among seniors.

\section{METHODS}

PlanYourLifespan.org

PlanYourLifespan.org (PYL) educates users on the health crises that often occur with age and connects them to posthospital and home-based resources available locally and nationally. PYL is personalized, dynamic, and adaptable in that all the information can be changed per the senior's wishes or changing health needs.

\section{Content of PYL}

Previously, we conducted focus groups with seniors about current and perceived home needs and aging-in-place. 
TABLE 1. Participant Baseline Characteristics (continued)

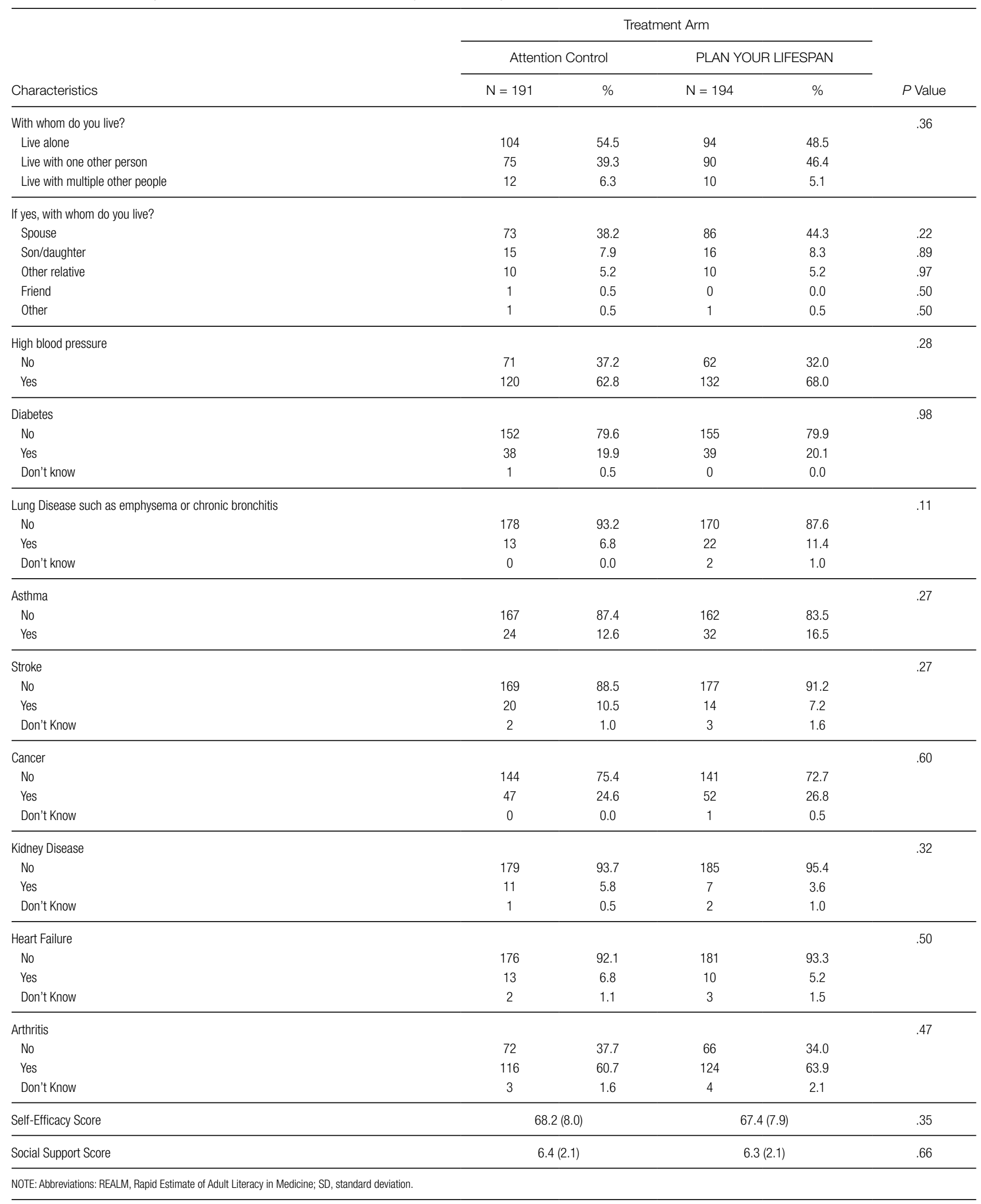


TABLE 2. Understanding of Posthospital Discharge and Home Services Over Time

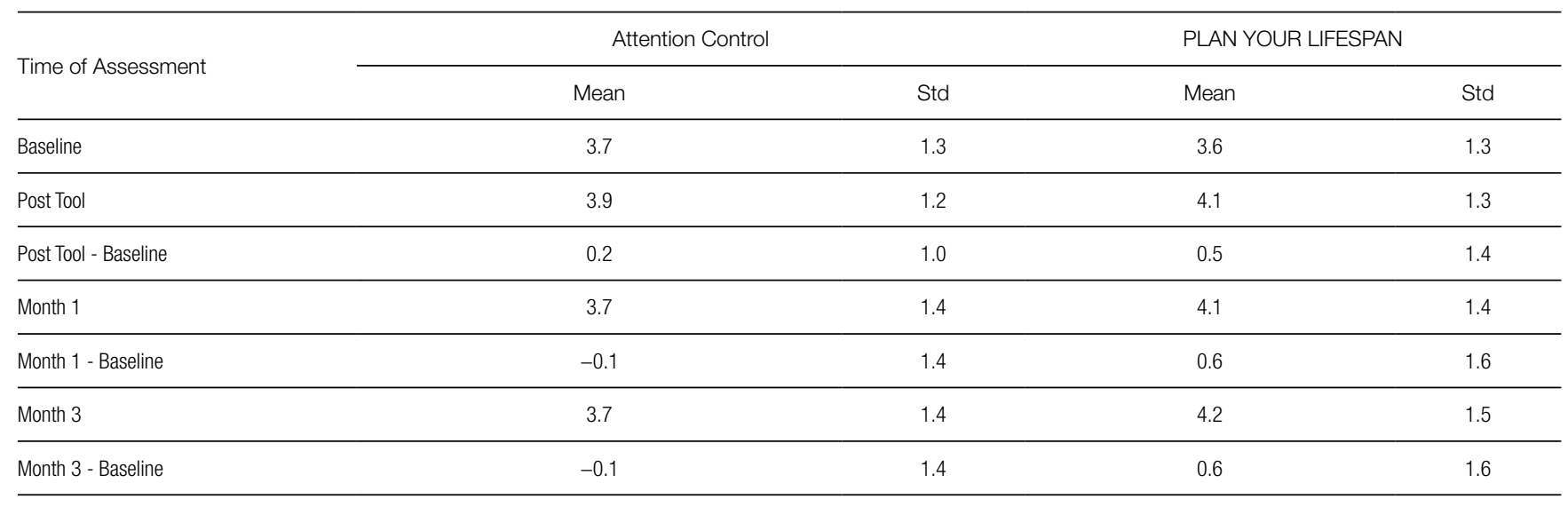

NOTE: Abbreviations: Std, standard deviation; UHS, Understanding of Posthospital Discharge and Home Services.

Major themes of what advanced life events (ALEs) would impact aging-in-place were identified as follows: hospitalizations, falls, and Alzheimer's. ${ }^{12}$ We organized PYL around these health-related ALEs. Our multidisciplinary team of researchers, seniors, social workers, caregiver agencies, and Area Agencies on Aging representatives then determined what information and resources should be included.

Each section of PYL starts with a video of a senior discussing their real-life personal experiences, with subsections providing interactive information on what seniors can expect, types of resources available to support home needs, and choices to be made. Descriptions of types of settings for therapy, options available, and links to national/local resources (eg, quality indicators for SNFs) are also included. For example, by entering their zip code, users can identify their neighborhood SNFs, closest Area Agency on Aging, and what home caregiver agencies exist in their area.

Users can save their preferences and revisit their choices at any time. To support communication between seniors and their loved ones, a summary of their choices can be printed or e-mailed to relevant parties. For example, a senior uses PYL and can e-mail these choices to family members, which can stimulate a conversation about future posthospital care expectations.

As inadequate health literacy and cognitive impairment are prevalent among seniors, PYL presents information understandable at all levels of health literacy and sensitive to cognitive load. ${ }^{9}$ There is simplified, large-font, no mouse scrolling and audio available for the visually impaired.

\section{Study Design and Randomization}

To test PYL, a 2-armed (attention control [AC] and PYL intervention), parallel, randomized controlled trial was conducted. Participants were randomly assigned to 1 of the 2 conditions via a pregenerated central randomization list using equal (1:1) allocation and random permuted block design to ensure relatively equal allocation throughout the study. The AC condition exposed participants to the $\mathrm{Na}$ - tional Institute on Aging-sponsored website, Go4Life.nia. nih.gov, an educational website on physical activity for seniors. This website has comparable design and layout to PYL but does not include information about advanced planning. The AC condition controlled for the possibility that regular contact with the study team may improve outcomes in participants randomized to the intervention website.

The trial was conducted from October 2014 to September 2015 in Chicago, Illinois; Fort Wayne, Indiana; and Houston, Texas. Inclusion criteria were as follows: age 65 and older, English-speaking, scoring $\geq 4$ questions correctly on the Brief Cognitive Screen, ${ }^{14}$ and current self-reported use of a computer or smartphone with internet. Participants were excluded if they had previously participated in the focus groups or beta testing of the PYL website. Community-based patient partners/stakeholders drove subject recruitment in their communities through word of mouth, e-mail bursts, newsletters, and flyers. At the Area Agency on Aging and community centers where services such as food vouchers and case management are provided, participants were recruited on-site and given study information. At the clinical sites, staff referred potential participants. Study materials such as flyers and information sheets were also located in the clinic waiting rooms. The Villages, nonprofit, grassroots, membership organizations that are redefining aging by being a key resource to community members wishing to age in place, heavily relied on electronic recruitment using their regular e-newsletters and e-mail lists to recruit potential participants. Potential subjects were also recruited by distributing flyers at local senior centers and senior housing buildings. Interested seniors contacted research staff who explained the study and assessed their eligibility. If eligible, subjects were scheduled for a face-to-face interview.

At the face-to-face encounter, all study subjects completed a written consent, answered baseline questions, and were randomized to either arm. Next, research staff introduced all study subjects to the website to which they were randomized and provided instructions on its use. Staff were present to 
TABLE 3. Linear Mixed Model Results for Evaluation of UHS over Time

\begin{tabular}{|c|c|c|c|c|}
\hline Models & Estimate & Lower 95\% CL & Upper 95\% CL & $P$ Value \\
\hline \multicolumn{5}{|l|}{ Unadjusted Model Output } \\
\hline Time & -0.04 & -0.11 & 0.03 & 0.22 \\
\hline Plan Your Lifespan Arm & -0.14 & -0.39 & 0.11 & 0.27 \\
\hline Time-by-Arm Interaction & 0.23 & 0.14 & 0.33 & $<.0001$ \\
\hline \multicolumn{5}{|l|}{ Adjusted Model Output } \\
\hline Time & -0.05 & -0.12 & 0.02 & 0.20 \\
\hline Plan Your Lifespan Arm & -0.14 & -0.39 & 0.10 & 0.24 \\
\hline Time-by-Arm Interaction & 0.22 & 0.12 & 0.32 & $<.0001$ \\
\hline Male Sex & -0.38 & -0.63 & -0.14 & 0.002 \\
\hline Income Level & 0.08 & 0.01 & 0.15 & 0.02 \\
\hline History of High Blood Pressure & -0.22 & -0.43 & -0.01 & 0.04 \\
\hline History of Kidney Disease & -0.51 & -0.96 & -0.06 & 0.03 \\
\hline Health Literacy Level & 0.38 & 0.12 & 0.63 & 0.004 \\
\hline Education Level & 0.20 & 0.06 & 0.34 & 0.004 \\
\hline
\end{tabular}

NOTE: Abbreviations: CL, PLEASE DEFINE ; UHS, Understanding of Posthospital Discharge and Home Services.

assist with questions as needed on navigation but did not assist with decision making for either website. A minimum of 15 minutes and a maximum of 45 minutes was allotted for navigating either website. After navigating their website, participants were administered an immediate in-person posttest survey. One month and 3 months after the faceto-face encounter, research staff contacted all study participants over the phone to complete a follow-up survey. Staff attempted to reach participants up to 3 times by phone. Data were entered into Research Electronic Data Capture survey software. ${ }^{15}$ This study was approved by the Northwestern University Institutional Review Board.

\section{Understanding of Posthospital Discharge and Home Services}

As part of the larger trial, which included behavioural outcomes that will be reported elsewhere, we sought to explore the effects of PYL on participants' knowledge and understanding of posthospital discharge and home services (UHS). Participants were asked to respond to 6 questions at baseline, immediate posttest, and at the 1- and 3-month follow-up time points. Knowledge items were developed by the study team in conjunction with the patient/partner stakeholders. UHS scores were calculated as the sum of the 6 questions (each scored 0 if incorrect and 1 if correct) with a possible range of 0-6.

\section{Covariates}

Demographic information, self-reported health, importance of religion, and existence of a power of attorney, living will, advanced directive (eg, Physician Orders for Life-Sustaining Treatment) were obtained via self-report. Participants were asked about their general and social self-efficacy using the validated Self-efficacy Scale ${ }^{16}$ and their social support using the Lubben Social Network Scale-6. ${ }^{17}$ Health literacy was assessed using the Rapid Estimate of Adult Literacy in Medicine-Short Form. ${ }^{18}$ To measure burden of disease, par- ticipant comorbidities were measured using a nonvalidated 9-item dichotomous response condition list, which included some items adapted from the Charlson Comorbidity Index and the Elixhauser Comorbidity Index.

\section{Statistical Analysis}

Data analysis included all available data in the intention-to-treat dataset. As UHS was collected at multiple time points up to 3 months postintervention, we employed linearmixed modeling with random participant effect and fixed arm, time, and time-by-arm interaction terms. The time-byarm interaction allows for comparison of UHS slopes (or trajectories) across arms. Analyses explored multiple potential covariates, including current utilization of services, physical function, comorbidities, social support, health literacy, self-efficacy, and sociodemographics. Those covariates found to have a significant association $(P<0.05)$ with outcome were considered for inclusion in the overall model selection process. Ultimately, we developed a final parsimonious, adjusted longitudinal model with primary predictors of time, arm, and their interaction, controlling for only significant baseline variables following a manual backward selection method. All analyses were conducted in SAS software (version 9.4, copyright 2012, The SAS Institute Inc., Cary, North Carolina).

\section{RESULTS}

Among 470 participants screened for eligibility, 385 were randomized (Figure). All were included in intention-to-treat analysis. Of the 191 participants allocated to the AC group, 1 participant partially received the PYL intervention. The mean age of participants was 71.9 (standard deviation = 5.6), and $79.5 \%$ were female; $62.9 \%$ identified as white and $37.1 \%$ as non-white (Table 1). Baseline characteristics were similar in both of the groups.

Table 2 presents follow-up summary statistics by arm for the UHS score. At both the 1- and 3-month time points, 


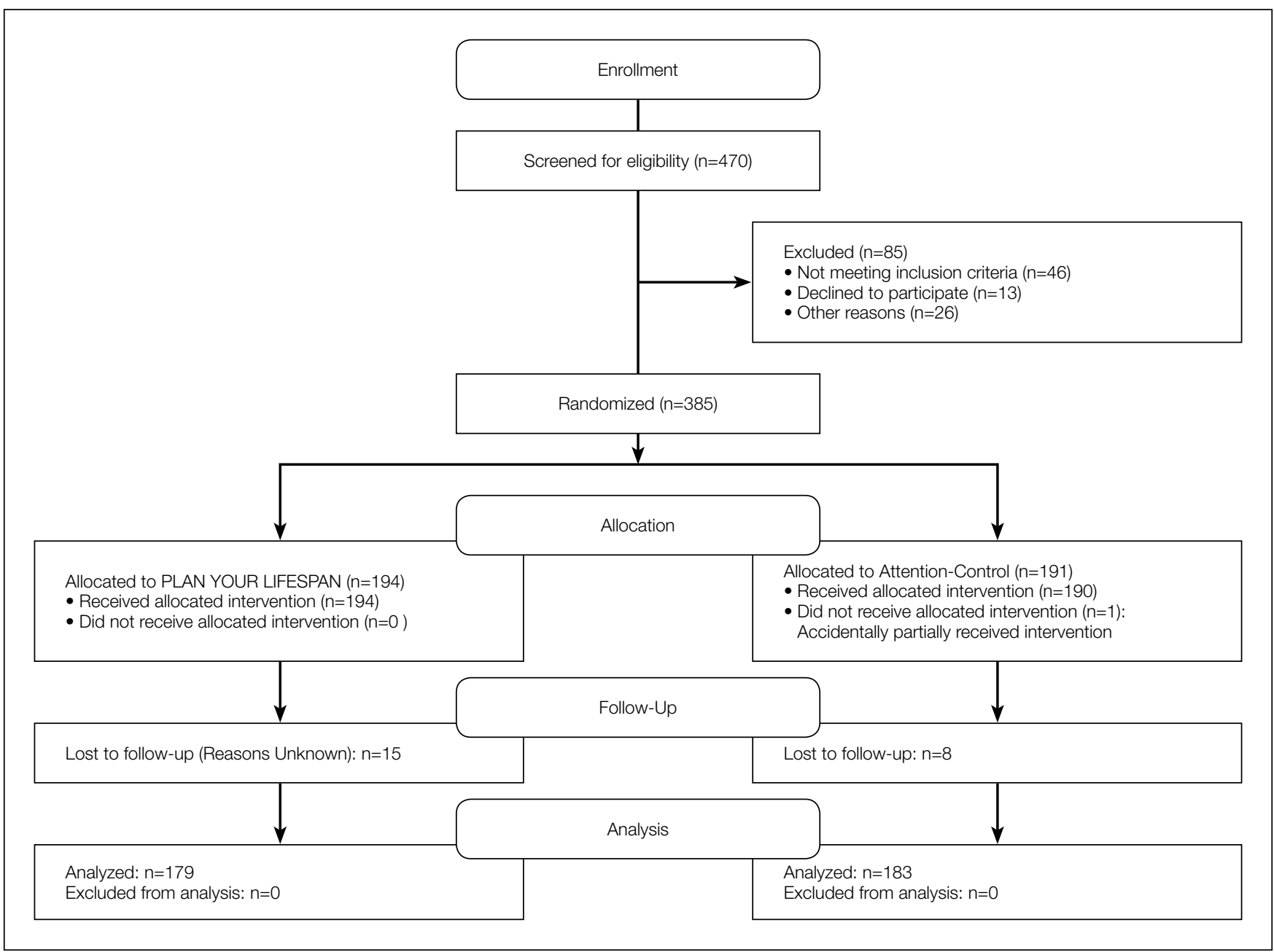

FIG. CONSORT diagram

the mean UHS score in the active intervention arm increased (by $0.56 \pm 1.55$ points at 1 month and $0.60 \pm 1.63$ at 3 months), while mean UHS score decreased in the AC arm at both time points $(-0.09 \pm 1.43$ at 1 month and $-0.07 \pm$ 1.37 at 3 months).

Table 3 illustrates linear mixed model results both failing to adjust and adjusting for potentially influential baseline covariates. In both instances, the interaction term (armby-time) was highly significant $(P<0.0001)$ in predicting UHS score, suggesting that, when compared to the AC arm, the intervention arm exhibited a large mean slope in UHS score over time. That is, understanding home services score tended to increase at a faster rate for those in the active arm. Higher levels of income $(P=0.0191)$, literacy $(P=0.0036)$, and education $(P=0.0042)$ were associated with increased UHS scores; however, male sex $(P=0.0023)$ and history of high blood pressure $(P=0.0409)$ or kidney disease $(P=$ 0.0278 ) were negatively associated with UHS scores.

\section{CONCLUSION/DISCUSSION}

The results of our study show that among seniors, PYL improved their understanding of home-based services and the services that may be required following a hospitalization. Educating seniors about what to expect regarding the transition home from a hospital before a hospitalization even occurs may enable seniors and their families to plan ahead instead of reacting to a hospitalization. PYL, a national, publicly available tool with links to local resources may potentially help in advancing transitional discharge care to prior to a hospitalization.

To our knowledge, this is one of the first websites and trials devoted to planning for seniors' health trajectory as they age into their 70s, 80s, 90s, and 100s. Clinicians regularly discuss code status and powers of attorney during their end-of-life discussions with patients. We encourage clinicians to ask patients, "What about the 10 to 20 years before you die? Have you considered what you will do if you get sick or need help at home?" While not replacing a social worker, the ability of PYL to connect seniors to local resources makes it somewhat of a "virtual social worker." With many physician practices unable to afford social workers, PYL provides a free-ofcharge means of connecting seniors to area resources.

A major strength of this project was our strong community partnerships. PYL was developed with significant input from 
our patient partners/stakeholders, which included seniors, senior community group leaders, Area Agencies on Aging, Villages, nurses, caregiver agency leaders, and clinicians. This patient and stakeholder engagement enabled us to create a website that was fully senior-centric, focusing specifically on what was important to seniors. Patient partners/ stakeholders were tasked with recruiting participants for the trial, using multiple tactics in their communities to connect potential participants to researchers. Recruiting directly through our community partners helped us include people who would not normally participate in research studies. An additional study strength was that recruitment occurred at multiple sites, including rural and urban locales.

As with all studies, limitations exist. While using a validated outcome measure would have been ideal for measuring knowledge, none existed that assessed whether a person understood their future needs or could plan to make use of available resources. Consequently, the UHS outcome measure was not validated prior to starting this trial and it remains unclear what changes in UHS score observed mean or translate to in a real-world setting.

The study participants were in general white, educated, and in reasonably good health. This may be a limitation of this study given that it could impact the generalizability of the study results, as we are unable to know for certain if these same results would be observed with participants who have lower educational levels and are in poor health. Power considerations in this study did not account for comparison of outcomes within specific subgroups so we were unable to assess outcomes in groups such as those with limited health literacy, low social support, or low self-efficacy. The trial was

\section{References}

1. Campbell SE, Seymour DG, Primrose WR, ACMEPLUS Project. A systematic literature review of factors affecting outcome in older medical patients admitted to hospital. Age Ageing. 2004;33:110-115.

2. Forster AJ, Clark HD, Menard A, et al. Adverse events among medical patients after discharge from hospital. CMAJ. 2004; 70:345-349.

3. Kane RL Finding the right level of posthospital care: "We didn't realize there was any other option for him”. JAMA. 2011;305(3):284-293.

4. Shepperd S, McClaran J, Phillips CO, et al. Discharge planning from hospital to home. Cochrane Database Syst Rev. 2010;(1):CD000313.

5. Horwitz LI, Moriarty JP, Chen C, et al. Quality of discharge practices and patient understanding at an academic medical center. JAMA Intern Med. 2013;173:1715-1722.

6. Creditor MC. Hazards of hospitalization of the elderly. Ann Intern Med. 1993;118(3):219-223.

7. U.S. Census Bureau, Population Division, Census 2003. https://www.census.gov/ programs-surveys/saipe/data/datasets.2003.html. Originally accessed September 1, 2016.

8. Russo, C. A., Elixhauser, A. Hospitalizations in the Elderly Population, 2003. Statistical Brief \#6. May 2006. Agency for Healthcare Research and Quality, Rockville, MD. http://www.hcup-us.ahrq.gov/reports/statbriefs/sb6.pdf. Accessed September 1, 2017.

9 U.S. Department of Health and Human Services, and U.S. Department of Labor. The future supply of long-term care workers in relation to the aging baby boom generation: Report to Congress. Washington, DC: Office of the Assistant Secretary for Planning and Evaluation, 2003. http:aspe.hhs.gov/daltcp/reports/ltcwork. htm. Accessed September 1, 2016. also limited by our inability to collect information on whether or not the knowledge gains observed in the study led to any measureable outcomes. Due to the relatively short follow-up time, we were unable to ascertain whether any study participants were hospitalized during the study follow-up period and if so, if exposure to PYL had any impact on patient anxiety, length of hospital stay, and/or caregiver burden. We were also unable to assess patients' ability to utilize and carry out their posthospitalization discharge plans if they had one in place. Future studies with longer follow-up are needed to determine these important, measurable outcomes.

Potential implications of planning for a senior's lifespan are expansive. If hospitalized seniors knew their preferred SNF for subacute rehabilitation on the first day of their hospitalization, hospital lengths of stay could potentially be reduced. If families knew which caregiver agencies, Area Agency on Aging, or Village their senior wished to use, obtaining services would perhaps be easier to accomplish.

\section{Acknowledgments}

This work was supported through a Patient-Centered Outcomes Research Institute Award (IH-12-11-4259). Dr. Lindquist and Dr. Ciolino had full access to all of the data in the study and take responsibility for the integrity of the data and the accuracy of the data analysis. Research reported in this publication was also supported, in part, by the National Institutes of Health's National Center for Advancing Translational Sciences, Grant Number UL1TR000150.

Disclaimer: All statements in this manuscript, including its findings and conclusions, are solely those of the authors and do not necessarily represent the views of the $\mathrm{Pa}$ tient-Centered Outcomes Research Institute, its Board of Governors or Methodology Committee, or the National Institutes of Health.

Disclosure: The authors have nothing to report.

10. The Henry J. Kaiser Foundation. Long-term Care: Medicaid's role and challenges [Publication \#2172]. Washington, DC: 1999.

11. AARP. Beyond 50.2003: A Report to the Nation on Independent Living and Disability, 2003, http://www.aarp.org/research/health/disabilities/aresearch-import-753.html. Accessed September 1, 2016.

12. Lindquist LA, Ramirez-Zohfeld V, Sunkara P, et al. Advanced Life Events (ALEs) that Impede Aging-in-Place among Seniors. Arch Gerontol Geriatrs. 2016;64: 90-95.

13. Doak CC, Doak LG, Root JH. Teaching Patients with Low Literacy Skills. Philadelphia, PA: JB Lippincott Co.; 1996.

14. Callahan CM, Unverzagt FW, Hui SL, Perkins AJ, Hendrie HC. Six-item screener to identify cognitive impairment among potential subjects for clinical research. Med Care. 2002;40:771-781.

15. Harris PA, Taylor R, Thielke R, Payne J, Gonzalez N, Gonzalez J, Conde JG. Research electronic data capture (REDCap) - A metadata-driven methodology and workflow process for providing translational research informatics support. J Biomed Inform. 2009;42(2):377-381.

16. Sherer M, Maddux JE, Mercandante B, Prentice-Dunn S, Jacobs B, Rogers RW. The Self Efficacy Scale: Construction and Validation. Psychol Rep. 1982;51(3):663-671.

17. Lubben JE. Assessing social networks among elderly populations. Fam Community Health. 1988;11(3):42-52.

18. Arozullah AM, Yarnold PR, Bennett CL, et al. Development and validation of the Rapid Estimate of Adult Literacy in Medicine. Medical Care. 2007;45(11): 1026-1033. 